\title{
Effective kaon energy from a novel chiral SU(3) model
}

\author{
Guangjun $\mathrm{Mao}^{1}$, P. Papazoglou ${ }^{1}$, S. Hofmann ${ }^{1}$, S. Schramm ${ }^{2}$, H. Stöcker ${ }^{1}$, and W. Greiner ${ }^{1}$ \\ ${ }^{1}$ Institut für Theoretische Physik der J. W. Goethe-Universität \\ Postfach 1119 32, D-60054 Frankfurt am Main, Germany \\ ${ }^{2}$ GSI Darmstadt, Postfach 1105 52, D-64220 Darmstadt, Germany
}

\begin{abstract}
A new chiral SU(3) Lagrangian is proposed to describe the properties of kaons and anti-kaons in the nuclear medium. The saturation properties of nuclear matter are reproduced as well as the results of the Dirac-Brückner theory. After introducing the coupling between the omega meson and the kaon, our results for effective kaon and anti-kaon energy are quite similar as calculated in the one-boson-exchange model.
\end{abstract}

The properties of kaons/anti-kaons in nuclear and neutron matter have attracted considerable interest. Substantial theoretical effort has been devoted to investigate medium effects on kaons and anti-kaons in dense matter [1, 2, 3, 4]. Recent data by the Kaos collaboration [5] on $K^{+}$and $K^{-}$ 7 production in relativistic heavy-ion collisions, which seems to exhibit a substantial enhancement of the $K^{-}$yield, stimulated further activity. Among the various models proposed, the chiral SU(3) Lagrangian seems to be particularly useful, since the kaon is essentially a pseudo-Goldstone boson. However, up to now, a consistent calculation based on a chiral Lagrangian, which can simultaneously describe both the kaon-nuclear interactions and the ground state of the dense matter, has not been performed yet.

This is the aim of the present work. It addresses the problem in a novel chiral SU(3)-symmetric Lagrangian [6]. In addition to the ground-state saturation properties of nuclear matter, the whole density dependence of the mean fields as predicted by the Dirac-Brückner theory [7] are considered as a further constraint to the model. This will turn out to be rather important for the investigation of the kaon and anti-kaon properties at higher densities. The chiral SU(3) Lagrangian reads

$$
\mathcal{L}=\mathcal{L}_{\text {kin }}+\sum_{W=X, V, u, \Gamma} \mathcal{L}_{B W}+\mathcal{L}_{v e c}+\mathcal{L}_{0}+\mathcal{L}_{S B}
$$

The main feature of the model is that the baryon masses are generated by the scalar condensates while their splitting is realized through SU(3) symmetry breaking for these condensates. The model is described in [6]. Considering SU(3) generators up to quadratic order, one can obtain the Lagrangian for nuclear matter ground state and kaon-nuclear interaction simultaneously [6, 8]. The kaon interaction is described by

$$
\mathcal{L}_{K N}=-\frac{3 i}{8 f_{K}^{2}} \bar{\psi} \gamma_{\mu} \psi \bar{K} \overleftrightarrow{\partial^{\mu}} K+\frac{m_{K}^{2}}{2 f_{K}}(\sigma+\sqrt{2} \zeta) \bar{K} K-i \mathrm{~g}_{\omega K} \bar{K} \overleftrightarrow{\partial^{\mu}} K \omega_{\mu}
$$

The omega-kaon coupling is introduced through considering the vector field as a gauge field. The parameters of the model are determined by the SU(3) vacuum and the saturation properties of nuclear matter. The corresponding saturation properties are: $m^{*} / m_{N}=0.641, E / A\left(\rho_{0}\right)=-15.93 \mathrm{MeV}$, 
$K=266.2 \mathrm{MeV}$ and $\rho_{0}=0.15 \mathrm{fm}^{-3} ; f_{\pi}=93.3 \mathrm{MeV}, f_{K}=122 \mathrm{MeV}, m_{\pi}=139 \mathrm{MeV}, m_{K}=498 \mathrm{MeV}$ The parameters of the kaon-nuclear interactions, Eq. (2), are constrained by the chiral Lagrangian itself. The model can reproduce the results of the G-matrix calculations [7] up to four times normal density [8]. After making a field shift to new variables $\phi$ and $\xi\left(\sigma=\sigma_{0}-\phi, \zeta=\zeta_{0}-\xi\right)$, we obtain the effective-mass and -energy of the kaon $K$ and the anti-kaon $\bar{K}$

$$
\begin{aligned}
& m_{K}^{* 2}=m_{K}^{2}+\frac{m_{K}^{2}}{2 f_{K}} \phi+\frac{m_{K}^{2}}{\sqrt{2} f_{K}} \xi, \\
& \omega_{K, \bar{K}}=\sqrt{m_{K}^{* 2}+\left(\frac{3}{8 f_{K}^{2}} \rho_{B}+\mathrm{g}_{\omega K} \omega_{0}\right)^{2}} \pm\left(\frac{3}{8 f_{K}^{2}} \rho_{B}+\mathrm{g}_{\omega K} \omega_{0}\right) .
\end{aligned}
$$

Fig. 1 depicts the kaon and anti-kaon effective energies calculated with Eqs. (3) and (4). The results of other models are also displayed for comparison. It can be seen that without the $\omega-K$ coupling our model predicts a rather weak potential for the anti-kaon compared to the predictions of other models. After introducing the $\omega-K$ coupling, the calculated effective energies for kaon and anti-kaon are quite similar as obtained in the one-boson-exchange model [2]. At normal density the optical potentials of kaon and anti-kaon are $U_{o p t}^{K}=21.53 \mathrm{MeV}$ and $U_{o p t}^{\bar{K}}=-98.7 \mathrm{MeV}$, respectively, which are in accordance with the data from the $K N$ scattering lengths and the results of other models [2, 4]. However, the $U_{o p t}^{\bar{K}}$ is still much weaker than the prediction from the $K^{-}$atomic data [9], which gives $U_{\text {opt }}^{\bar{K}}=-200 \pm 20$ $\mathrm{MeV}$. Further investigation is needed.

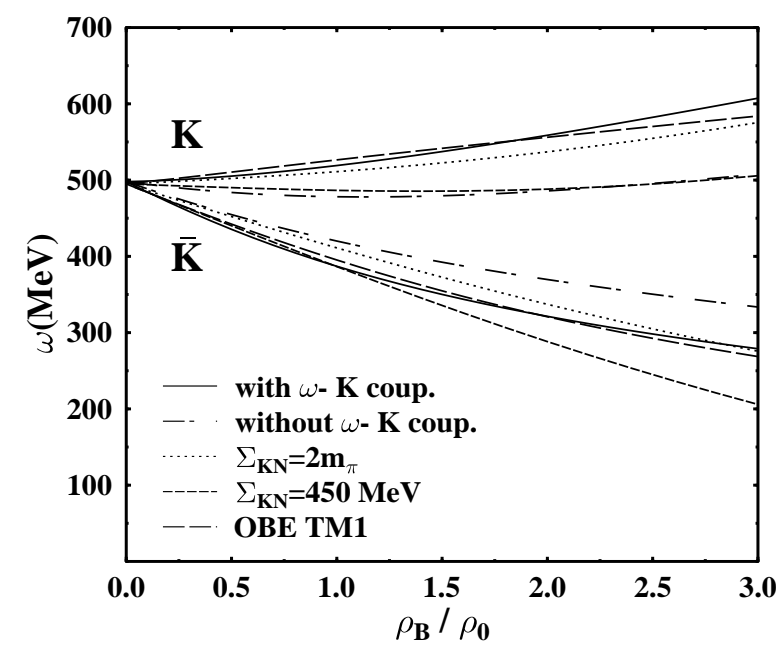

Fig. 1: The energies of kaons and anti-kaons as a function of the density. The solid and dot-dashed line represent the results of this work with and without the $\omega-K$ coupling, respectively. The dotted and short dashed line are calculated with chiral perturbation theory with the different $K N$ sigma terms. The long dashed line depicts the results of one-bosonexchange model with the TM1 parameter set.

\section{References}

[1] G.E. Brown et al., Z. Phys. A341 (1992) 301; Nucl. Phys. A567 (1994) 937.

[2] J. Schaffner et al., Phys. Lett. B334 (1994) 268; Phys. Rev. C53 (1996) 1416; Nucl. Phys. A625 (1997) 325.

[3] V. Koch Phys. Lett. B337 (1994) 7.

[4] T. Waas et al., Phys. Lett. B365 (1996) 12; Phys. Lett. B379 (1996) 34; Nucl. Phys. A594 (1995) 325.

[5] R. Barth et al., Phys. Rev. Lett. 78 (1997) 4007.

[6] P. Papazoglou et al., Phys. Rev. C57 (1998) 2576; nucl-th/9806087, Phys. Rev. C in press.

[7] R. Brockmann and R. Machleidt, Phys. Rev. C42 (1990) 1965.

[8] Guangjun Mao et al., nucl-th/9806068.

[9] E. Friedman, A. Gal, and C.J. Batty, Phys. Lett. B308 (1993) 6; Nucl. Phys. A579 (1994) 518. 